\title{
Disease mechanism, biomarker, and therapeutics for spinal and bulbar muscular atrophy (SBMA)
}

Atsushi Hashizume ${ }^{1 *}$, Kenneth H. Fischbeck ${ }^{2}$, Maria Penuto ${ }^{3,4,5,6}$, Pietro Fratta ${ }^{7}$, Masahisa Katsuno ${ }^{1 *}$

1. Department of Neurology, Nagoya University Graduate School of Medicine, Nagoya 466-8550, Japan

2. National Institute of Neurological Disorders and Stroke, National Institutes of Health, Bethesda, MD, USA

3. Department of Biomedical Sciences (DBS), University of Padova, 35131, Padova, Italy

4. Veneto Institute of Molecular Medicine (VIMM), Padova 35100, Italy

5. Myology Center (Cir-Myo), University of Padova, 35100 Padova, Italy

6. Padova Neuroscience Center (PNC), 35100 Padova, Italy

7. Department of Neuromuscular Diseases, UCL Queen Square Institute of Neurology, Queen Square, WC1N 3BG, London, UK

\section{*Correspondence may be sent to Dr Hashizume or Katsuno}

Atsushi Hashizume, MD, PhD

Department of Neurology, Nagoya University Graduate School of Medicine

65 Tsurumai-cho, Showa-ku, Nagoya 466-8550, Japan

Tel: +81-52-744-2391, Fax: +81-52-744-2394

E-mail: hassy0707@med.nagoya-u.ac.jp

Masahisa Katsuno, MD, PhD

Department of Neurology, Nagoya University Graduate School of Medicine

65 Tsurumai-cho, Showa-ku, Nagoya 466-8550, Japan

Tel: +81-52-744-2385, Fax: +81-52-744-2384

E-mail:ka2no@med.nagoya-u.ac.jp 
Word count: 171 words in abstract; 5666 words in text (including references).

Number of figures: 2 figures.

Number of tables: 1 table.

Key words: spinal and bulbar muscular atrophy; androgen receptor; polyglutamine; motor neuron; skeletal muscle; biomarker; animal model; clinical trial

Contributors: AH, MP, PF, KF, and MK drafted, reviewed, and edited the manuscript.

Funding: This work was funded by JSPS KAKENHI Gran Numbers JP17H04195 and JP20H00527 (to MK); a grant from the Japan Agency for Medical Research and Development (AMED) (No. 19ek0109359h0002 to MK), a grant from the Hori Sciences and Arts Foundation (to MK), Telethon-Italy (GGP19128 to MP), Association Française contre les Myopathies (22221 to MP), CNCCS Scarl Pomezia (to MP), and NIH-R21 (1R21NS111768-01 to MP), UK Medical Research Council (to PF), The Motor Neurone Disease Association (to PF), the NIHR UCLH Biomadical Research Centre (to PF), The Neuro Reserch Trust (to PF), The Rosetrees Foundation (to PF), and intramural research funds from the National Institute of Neurological Disorders and Stroke (to KF).

Competing interests: AH is supported by a JSPS KAKENHI Grant Number JP18K07523. PF has received honoraria from Nido Biosciences. MK is supported by a JSPS KAKENHI Grant Number JP17H04195, grants from the Japan Agency for Medical Research and Development (Nos. 19ek0109221h0003, 19ek0109359h0002, 19dk0207027h0004, 191k0201101h0001 and 19dm0107155h0001), and a grant from the Hori Sciences and Arts Foundation. He received honoraria from Takeda Pharmaceutical Co. Ltd., Alnylam Japan, Daiichi Sankyo Co. Ltd., Otsuka Pharmaceutical Co. Ltd., Novartis Pharma Co. Ltd., Biogen Japan and UCB Japan and grants from Zenyaku Kogyo Co. Ltd., Japan Blood Products Organization, Mitsubishi-Tanabe Pharma, CSL Behring Co. Ltd., Dainippon Sumitomo Pharma Co. Ltd., Otsuka Pharmaceutical Co. Ltd. and Daiichi Sankyo Co. Ltd.

Patient consent for publication: Not required. 


\begin{abstract}
Spinal and bulbar muscular atrophy (SBMA) is a hereditary neuromuscular disorder caused by CAG trinucleotide expansion in the gene encoding the androgen receptor $(A R)$. In the central nervous system, lower motor neurons are selectively affected, whereas pathology of patients and animal models also indicates involvement of skeletal muscle including loss of fast-twitch type 2 fibers and increased slow-twitch type 1 fibers, together with a glycolytic-to-oxidative metabolic switch. Evaluation of muscle and fat using MRI, in addition to biochemical indices such as serum creatinine level, are promising biomarkers to track the disease progression. The serum level of creatinine starts to decrease before the onset of muscle weakness, followed by the emergence of hand tremor, a prodromal sign of the disease. Androgen-dependent nuclear accumulation of the polyglutamine-expanded AR is an essential step in the pathogenesis, providing therapeutic opportunities via hormonal manipulation and gene silencing with antisense oligonucleotides. Animal studies also suggest that hyperactivation of Src, alteration of autophagy, and a mitochondrial deficit underlie the neuromuscular degeneration in SBMA, and provide alternative therapeutic targets.
\end{abstract}




\section{Introduction}

Spinal and bulbar muscular atrophy (SBMA) is a hereditary neuromuscular disorder which has several facets from a mechanistic point of view. First, SBMA is one of neurodegenerative disorders including Huntington's disease, caused by exonic trinucleotide repeat expansion, and it likely has pathophysiological overlap with other repeat expansion diseases. Second, within the central nervous system the disease primarily affects lower motor neurons, implying mechanistic commonalities with other motor neuron diseases including amyotrophic lateral sclerosis (ALS). Third, increasing evidence indicates involvement of both motor neuron and skeletal muscle in animal models as well as in patients, suggesting degeneration of multiorgan network in SBMA. Lastly, but not least, the monogenic and slowly progressive nature of the disease provides a wide range of opportunities for developing disease-modifying and, hopefully, preventive therapies. In this review, we summarize recent progress in molecular and clinical researches on disease mechanism, biomarker, and therapies of SBMA.

\section{Clinical phenotype}

SBMA is sex-linked and manifests in adult males with slow progression. The prevalence is estimated to be 1-2 per 100,000, whereas some patients may have been misdiagnosed with other neuromuscular diseases such as ALS. ${ }^{1}$ The principal symptoms are weakness and atrophy of bulbar and extremity muscles mainly attributable to lower motor degeneration in the brainstem or spinal cord accompanied by primary muscle involvement. Muscle weakness tends to be predominant in proximal muscles, and about $70 \%$ of the patients first notice weakness in their lower limbs (Fig. 1). Postural hand tremor usually occurs more than ten years before the onset of muscle weakness. Deep tendon reflexes are diminished or absent with no pathological reflexes. Sensory involvement is largely restricted to vibration sense, which is affected distally in the legs. Contraction fasciculations, which likely result from a remnant of large voluntary motor unit potentials, are especially noticeable in face and tongue. Cerebellar symptoms are absent. Dysarthria is common, with hypernasality due to incomplete lifting of soft palate. Patients sometimes experience laryngospasm, a sudden sensation of dyspnea. Swallowing dysfunction is characterized 
by impaired tongue movement at the oral phase, and nasal penetration followed by pharyngeal residue. At the advanced stage, swallowing dysfunction frequently results in aspiration pneumonia. More than half of patients die from respiratory infectious diseases. ${ }^{2}$ In addition to the neurological dysfunction mentioned above, patients with SBMA show androgen insensitivity including gynecomastia, testicular atrophy, erectile dysfunction, and decreased fertility. Blood testing shows elevated serum levels of creatine kinase $(\mathrm{CK})$ and liver enzymes, decreased serum creatinine, and glucose intolerance even before the neurological onset. ${ }^{3,4}$

Currently, there is no definitely effective treatment for SBMA; however, some disease-modifying drugs have been tested, based on the results of basic research to elucidate the disease mechanism of SBMA (Table 1). A phase 2 randomised control trial of leuprorelin acetate, a luteinising hormone-releasing hormone agonist, in which a total of 50 patients were enrolled, showed decreased accumulation of mutant AR protein in the scrotal skin and potential benefits for swallowing function. ${ }^{5}$ Thirty-six out of 50 subjects who participated in the phase 2 clinical trial continued to undergo administration of leuprorelin acetate in a following open-label trial. Compared with the natural history data obtained from 29 patients with SBMA who had never undergone disease-specific treatment, the patients treated with leuprorelin acetate showed a slower decline of motor functional scales such as the Revised Amyotrophic Lateral Sclerosis Functional Rating Scale (ALSFRS-R). Event-free survival was better in the treated group, suggesting the long-term disease modification of SBMA by leuprorelin acetate. ${ }^{6}$ However, the benefit of this therapy was not clearly shown in the subsequent multicenter phase 3 trial in a total of 204 subjects. ${ }^{7}$ Similarly, dutasteride, a 5-alpha reductase inhibitor that decreases levels of dihydrotestosterone, failed to show an effect on the primary outcome measure (quantitative muscle testing) in a phase 2 clinical trial, although there were secondary benefits on physical quality of life and the frequency of falls. ${ }^{8}$ As for other therapies, clenbuterol improved walking capacity, and an IGF-1 mimetic increased muscle volume in phase 2 clinical trials, although their efficacy has not been clearly shown in a large-scale trial. ${ }^{9}{ }^{10}$ Possible reasons for failure in clinical trials may include lack of established outcome measures, variety of severity and progression, relatively long preclinical phase, and adverse effects of tested 
drugs.

\section{Biomarkers}

Biomarkers are biological properties or molecules that can be measured, and broadly fall under three categories and purposes: a) diagnostic; b) prognostic; c) markers of disease progression. The monogenic nature of SBMA and clear genetic diagnosis currently make novel diagnostic markers not a strong priority. Conversely, as outlined above, SBMA disease progression is variable, and therefore tools to predict disease course would be beneficial for patient knowledge and also for stratification in clinical trials - no such markers are currently available. In this review, we will focus on biomarkers of disease progression. SBMA is a slowly progressive disease and markers that are able to detect and quantify progression over 12 months are strongly needed in order to allow for effective, brief and small clinical trials. Progression biomarkers range from quantifying muscle function and force, to assessing the neuromuscular status by neurophysiology and imaging, and finally to measuring molecules in blood to assess disease activity and progression.

Function rating scales, a number of which were developed for other neuromuscular conditions, are currently used in SBMA clinical practice and have been incorporated in clinical trials. ${ }^{11}$ Amongst these, is worth highlighting the SBMA Functional Rating Scale (SBMAFRS), a 14-item scale specifically adapted to SBMA, which covers bulbar, upper and lower limb, trunk, and respiratory aspects of disease. ${ }^{12}$ Function rating scales are very simple tools, extremely useful to rapidly assess the degree of disease impact, but lack the sensitivity and reliability to detect changes over 12 month periods of time.

More time consuming, but also more sensitive than function rating scales, are functional assessments. Video fluorography detects bulbar function changes over 12 months and was successfully used as a primary outcome measure in clinical trials. ${ }^{7}$ The six-minute walk test (6MWT) is a simple and effective measure of lower limb function, and the adult myopathy assessment tool (AMAT) assesses limb and trunk function, with a focus on repeat exercise and endurance - both 6MWT and AMAT 
have been used in clinical trials and shown to detect disease changes over 12 and 24 months, respectively, as mentioned above. ${ }^{8-10} \mathrm{~A}$ limit of both tools is the inability to capture changes in advanced patients, who are unable to undergo these assessments. Muscle strength can be directly quantified through a range of myometry approaches, where generally portability, cost and ease of measurement inversely correlate with reproducibility. Limb myometries can detect changes over 18 months, ${ }^{13}$ whilst tongue pressure has also been assessed and shown encouraging results by correlating with bulbar function and the ability to detect changes before subjective awareness of dysphagia. ${ }^{14}$

In recent years, there has been increasing interest and excitement across neuromuscular conditions for the use of skeletal muscle MRI to track disease changes in a sensitive, quantitative and operator-independent manner. ${ }^{10}$ Muscle MRI can indeed allow assessment of muscle size, and also quantify muscle fat infiltration through Dixon acquisition approaches. Similarly to what is observed both in myopathies and in chronic denervation disorders, muscle fat infiltration is also a prominent feature in SBMA, and occurs in both limb and bulbar muscles. Interestingly, initial MRI studies have documented a specific stereotypical pattern of muscle involvement in SBMA, with certain muscles, such as the tibialis anterior and posterior, and the sartorius being relatively spared from degeneration. Most importantly, fat infiltration correlates with disease functional involvement, and changes in fat infiltration over 18 months demonstrated this technique is sensitive in detecting progression, making it a very strong candidate to be used as an outcome measure. ${ }^{13,15}$ Future studies to determine changes on shorter time periods and inter-centre reproducibility will be useful in optimizing its use in clinical trials.

Neurophysiology approaches have also been applied in SBMA, and motor unit number estimations (MUNE) were shown to correlate with function and detect disease progression over 12 months. ${ }^{16,17}$ Improvements in estimation of motor units have emerged over the last decade, and need to be assessed in SBMA.

The measurement of molecules in biofluids can be effective in documenting disease 
activity, and the use of blood-derived biofluids is extremely appealing because of the low invasiveness required, and the possibility of conducting multiple measurements over time. A number of conventional metabolic and liver function markers are often altered in SBMA, documenting the non-neurological involvement of disease, but we will here focus on changes that can serve as biomarkers of the neuromuscular component of disease. Neurofilament (NF), both light chain (NfL) and phosphorylated heavy chain $(\mathrm{pNfH})$, can be accurately measured in serum or plasma and have been shown to increase across a number of neurological disorders ranging from the central nervous system (CNS) acute and chronic diseases to slowly progressive peripheral nervous system (PNS) diseases such as CMT, and also to correlate with disease progression rate in ALS. Intriguingly, NF changes were not detected in SBMA patients and mouse models of disease, owing possibly to a very slow degeneration of neurons during disease. ${ }^{18}$ Future studies exploring PNS specific markers, such as peripherin, will be important to try and establish markers of neuron degeneration in SBMA.

Muscle degeneration due to both a primary myopathic component and denervation is a prominent feature of SBMA, and indeed CK, a marker of muscle damage, are elevated in SBMA. Unfortunately, increased CK are variable when measured longitudinally and are not very informative of disease progression and stage. ${ }^{15}$ Conversely, creatinine levels reflect muscle mass and decrease with disease progression in a number of neuromuscular conditions. Muscle uptake of creatinine has been shown to be impaired in SBMA, making its reduction in plasma levels more accentuated. Importantly, creatinine decrease does correlate with disease progression stages. ${ }^{18,19}$ Further markers able to determine the level of neuronal and muscle disease activity will be beneficial for SBMA, and potentially offer the opportunity to obtain early information on treatment efficacy.

In summary, functional and muscle strength assessments, blood-based biomarkers and skeletal muscle imaging do currently allow to monitor disease progression and should be used in combination in clinical trials.

\section{Genetics}


SBMA is caused by a CAG repeat expansion in the $A R$ gene on the $\mathrm{X}$ chromosome at q11-12, which leads to an expanded polyglutamine tract in the N-terminal domain of the AR protein. ${ }^{20}$ SBMA was the first of many neurological disorders now known to be caused by repeat expansion, including at least nine that are caused by expanded polyglutamine tracts.

In healthy individuals the repeat is polymorphic, with a range of about 11-36 CAGs, and in SBMA patients it is expanded to 39-72 CAGs. Recently, repeat lengths up to 39 CAGs were reported in a population sample of over 14,000 individuals in northern Europe at a frequency greater than the estimated disease prevalence, suggesting underdiagnosis or mitigating factors leading to reduced penetrance. ${ }^{21}$ At the high end of the disease range clinical features of abnormal genital development and dilated cardiomyopathy have been reported. ${ }^{22,23}$ There is evidence of a founder effect in the Japanese population, where the normal repeat length distribution is somewhat higher, and clusters have also been reported in western Finland and northern Italy.

The repeat is modestly unstable, with expansions of contractions of a few CAGs with transfer from one generation to the next, and there is a correlation of repeat length with age of onset and disease severity, such that the longer the repeat the earlier the onset and the more severe the disease manifestations. ${ }^{20}$ There is also evidence of repeat length variation in sperm but not somatic cells within individuals. The phenomenon of anticipation, in which subsequent generations have an earlier onset and more severe symptoms due to expansion of CAG repeat length, is not usually observed in SBMA. ${ }^{24}$

The AR is a nuclear receptor, and the $\mathrm{N}$-terminal domain is involved in interaction with other nuclear proteins that regulate transcription of target genes. Although the mutation causes some loss of normal AR function, as evidenced by the signs of androgen insensitivity that occur in SBMA patients, the primary effect of the mutation is to cause a toxic gain of function in the receptor protein, i.e., it alters the protein structure such that the receptor becomes toxic to motor neurons and muscle. This is based on the finding that other mutations that cause loss of AR function result in a different phenotype, with feminization but not the progressive weakness and motor neuron loss 
of SBMA. Further support for a gain of function comes from the finding that the mutant protein is toxic and reproduces features of the disease phenotype in cultured cells and transgenic animals, as discussed below.

\section{Androgen-dependent disease mechanism}

The causative protein of SBMA, AR, is a nuclear receptor belongings to the steroid/thyroid hormone receptor family. AR mediates the effects of androgens, testosterone and dihydrotestosterone, through binding to an androgen response element in the target genes and thereby facilitating their expression.

Nuclear accumulation of the polyglutamine-expanded AR is not only the cardinal histopathological feature, but also an essential step in the degenerative process in SBMA (Fig. 2). Without androgen stimulation, AR remains in the cytoplasm, being kept inactive by heat shock proteins and other molecular chaperones. The receptor requires ligand binding for dissociation from chaperones, interdomain $\mathrm{NH} 2$ - and carboxyl-terminal (N/C) interaction, translocation into the nucleus, dimerization, binding to DNA, and transcriptional activation, as well as instigation of non-DNA binding-dependent signaling. ${ }^{25}$ The ligand-dependent nuclear translocation of AR appears to play an essential role in the disease mechanism of SBMA. The phenotypic difference with gender, which is a specific feature of SBMA, has been recapitulated in animal models. In a transgenic mouse model of SBMA expressing the full-length human AR containing 97 CAGs (AR-97Q), neuromuscular symptoms are markedly pronounced and accelerated in the male mice, and androgen deprivation through surgical or chemical castration substantially improved the symptoms and histopathological findings by suppressing nuclear accumulation of the polyglutamine-expanded AR. ${ }^{26}$ This provides a theoretical basis for development of ligand modulating therapies including leuprorelin acetate for SBMA.

In addition to nuclear translocation, ligand-dependent N/C interaction is requisite of toxicity of the polyglutamine-expanded AR. Inhibition of the interdomain interaction via genetic manipulation suppresses aggregation of the polyglutamine-expanded AR and thereby mitigates motor deficits in a transgenic mouse model of SBMA. ${ }^{27}$ Similar 
beneficial effects on SBMA pathogenesis were also exerted by small molecules that stabilize interaction between Hsp70 and N-terminal domain of AR. ${ }^{28}$ Furthermore, a mutation that inhibits the binding of the polyglutamine-expanded AR to DNA without affecting ligand binding abolishes neurodegeneration, indicating that DNA binding is also necessary for the toxicity. ${ }^{29}$ The ligand-dependent interactions between the carboxyl-terminal AF-2 domain of AR and co-regulators are also required for the pathogenesis of SBMA. ${ }^{29}$ AF-2 modulators inhibit aggregation of the polyglutamine-expanded AR, resulting in attenuation of neuromuscular degeneration in a transgenic mouse model of SBMA in which the pathogenic AR is expressed at an endogenous level. ${ }^{30}$ Post-translational modifications of AR including phosphorylation have also been implicated in the neurotoxicity of the polyglutamine-expanded AR. ${ }^{1}$

Inclusions of mutant AR are a histopathological hallmark of SBMA and found in both the nucleus and the cytoplasm in different cells and tissues from SBMA patients and animal models. ${ }^{31}$ It is also known that aggregation of the pathogenic AR occurs in a ligand-dependent manner. ${ }^{26}$ There is, however, a limited knowledge about how aggregated AR induces dysfunction and death of motor neuron and myocyte, but it has been shown that the pathogenic AR induces similar changes of intracellular signaling in both types of cells. For instance, the pathogenic AR with elongated polyglutamine activates Src, a non-receptor protein tyrosine kinase, both in the spinal cord and skeletal muscle of AR-97Q mouse. ${ }^{32}$ An Src inhibitor ameliorates the neuromuscular phenotype of SBMA by mitigating Src-dependent activation of p130Cas, without suppressing the nuclear accumulation of the pathogenic AR. In addition, the polyglutamine-expanded AR causes epigenetic dysregulation such as histone deacetylation and DNA hyper methylation in neurons. ${ }^{33}$

\section{Muscle pathogenesis}

Dysfunctions of each component of the motor unit result in a plethora of pathological conditions, from motor neuron diseases to peripheral neuropathies, myasthenia, and myopathies. Because the motor neuron and the innervated muscle fibers are in a tight structural and trophic relationship with each other, cell-autonomous degeneration of the motor neuron may cause noncell-autonomous degeneration of the innervated fiber and 
vice-versa. This is the case of SBMA. SBMA muscle biopsy pathological analysis reveals signs of denervation, with atrophic, grouped and angulated fibers, and myopathy, including presence of fibers with central nuclei, fiber degeneration, necrosis, macrophage infiltration, thus supporting that idea that cell-autonomous pathological processes occur not only in motor neurons, but also in myofibers. ${ }^{34,35}$ SBMA muscles show a fiber-type switch with loss of fast-twitch type 2 fibers and increased number of slow-twitch type 1 fibers, together with a glycolytic-to-oxidative metabolic switch. ${ }^{36,37}$ A key feature of SBMA muscle pathology is the presence of hypertrophic fibers, which further suggest a myopathic picture coexisting with neurogenic atrophy. ${ }^{34,35,38}$ Although not denervated, neuromuscular junctions show morphologic abnormalities and instability, which may hamper neurotransmission. ${ }^{39}$ Moreover, patients show reduced muscle contractility, weakness and increased fat content. ${ }^{40}$ Although there is no doubt that SBMA is an androgen-dependent disease, muscle strength positively correlates with serum androgen levels, implying that this aspect of disease may be the result of diminished response to circulating androgens. ${ }^{41}$ Fiber atrophy and hypertrophy, type IIb-to-IIa/IIx and glycolytic-to-oxidative fiber-type switch are disease manifestations well recapitulated in animal models of SBMA. ${ }^{37}$ In SBMA mice fast-twitch muscles are more severely affected compared to slow-twitch muscles and most of the pathological manifestations occur in muscle before spinal cord. ${ }^{37}$ Analysis of muscle pathology in presymptomatic subjects may clarify whether myopathic signs precede neurogenic atrophy in patients also. Another important finding that recently emerged from analysis of the phenotype of a new conditional mouse model of SBMA is that expression of mutant AR starting at about 40 days of age is sufficient to induce muscle atrophy and motor dysfunction, but not the fiber-type switch and metabolic alterations, thereby suggesting that expression of mutant AR during development is important to elicit these aspects of muscle pathology, at least in the mouse. ${ }^{42}$

Mechanistically, pathological processes occurring in SBMA muscle involve hyperactivation of protein kinase B (also known as Akt) and mammalian target of rapamycin (mTOR) signaling, which leads to increased protein synthesis, and concomitant activation of atrogenes and autophagy genes. ${ }^{37}$ Rapamycin treatment 
exacerbates the phenotype, supporting the idea that activation of Akt/mTOR signaling is a compensatory pathway to protein breakdown. However, activation of anabolic pathways is not sufficient to compensate activation of atrophy pathways and results in decreased net protein content in muscle. Importantly, expression of several autophagy genes is enhanced, a process that is likely to involve transcription factor EB (TFEB), but accumulation of lipidated microtubule-associated protein 1A/1B-light chain 3 (LC3) and sequestosome 1 (Sqstm1, p62) and the presence of autophagosomes reveals that the autophagy flux is altered in SBMA muscle. ${ }^{37,42}$

Another important aspect of muscle pathology concerns the status of mitochondria. Indeed, the expression of the oxidative phosphorylation enzymes is altered, and mitochondria are depolarized in the muscle of SBMA mice. ${ }^{37,42}$ Importantly, in muscle biopsy specimens of SBMA patients, muscle fibers have central core-like features, with reduced or absent mitochondria in the center of the fiber, and mitophagy. ${ }^{38}$ Interestingly, the polyglutamine-expanded $\mathrm{AR}$ is found in association with mitochondria. ${ }^{38,46}$ Reduced muscle contractility is associated with altered calcium dynamics, ${ }^{47}$ and aberrant expression of excitation-contraction coupling genes both in mice and patients. Altered expression of these genes may be either an indirect consequence of aberrant function of epigenetic reader and writer factors, or direct binding of mutant AR to the promoters and enhancers of these genes, which indeed have putative androgen-responsive elements in their core promoters and regulatory regions. This observation implies a physiological role of AR in the control of genes involved in muscle contraction and in the pathological processes occurring in SBMA. Skeletal muscle represents a good therapeutic target tissue for several reasons. Skeletal muscle is about $30-40 \%$ of our body weight and controls body metabolism. Therefore, any pathological process leading to alteration of muscle metabolism is expected to impact adipose tissue and liver, and whole-body metabolism. Consistent with the idea that muscle is a primary target tissue for therapy development, genetic and pharmacological inhibition of AR expression in muscle either prevented or ameliorated the phenotype of several mouse models of disease. ${ }^{48-50}$

\section{Therapy targeting the CNS}


Although there is considerable evidence of muscle involvement in SBMA, the disease is primarily caused by motor neuron degeneration. Clinical manifestations include muscle atrophy and fasciculations, which are signs of denervation and motor neuron dysfunction. Electrophysiologic MUNE studies show loss of motor neurons. ${ }^{16,17} \mathrm{As}$ mentioned above, SBMA patients are often initially diagnosed as having ALS, and ALS registries and natural history studies have included SBMA patients by mistake. ${ }^{41}$ Autopsies have demonstrated loss of motor neurons in the anterior horn of the spinal cord and in the brainstem. ${ }^{51}$ In cell culture and animal models, too, there is evidence of neuronal vulnerability and motor neuron loss.

Probably the best therapeutic approach for SBMA is reducing levels of the toxic AR protein, since this would prevent all its downstream deleterious effects. Reduction of AR expression in the CNS and muscle with a miRNA targeting the AR mRNA resulted in benefits in SBMA transgenic mice. Targeted antisense oligonucleotide (ASO) therapy has been remarkably effective for spinal muscular atrophy, and clinical trials in patients with familial ALS and Huntington's disease are also showing promise. A similar approach has shown preclinical efficacy for SBMA. In three different SBMA mouse models ASO suppression of AR mRNA systemically or in the CNS has been beneficial, with dose-responsive clearance of high molecular weight insoluble species, sustained reduction in soluble AR, and clearance of mutant AR pathology. ${ }^{52,53}$ ASO administration into the cerebral ventricles and target engagement in spinal cord has beneficial effects on motor neuron and neuromuscular junction pathology. ${ }^{53}$ The knock-in mouse model used for studies of peripheral suppression does not have the motor neuron loss that occurs in SBMA patients, and the cause of death is urinary retention (probably due to autonomic dysfunction), which is minimal in humans. Reducing mutant AR in the CNS alone would avoid the anti-anabolic effects of androgen reduction in peripheral tissue, which may have limited the benefits of androgen ligand reduction in clinical trials. Importantly, there is a clear path forward for intrathecal CNS ASO delivery, based on clinical trial results with other motor neuron diseases, while delivery of currently available ASOs to muscle at adequate levels in patients has been challenging. Thus, the rationale for CNS AR suppression with a targeted ASO is strong. 


\section{Other candidate therapies}

At least 30 different therapeutic approaches have shown efficacy in SBMA mice. In addition to RNA-based approaches such as ASO and RNA interference, viral delivery of miR-196a, which enhances the decay of the AR mRNA by silencing CELF2, a protein involved in regulation of AR mAR stability, decreases the protein level of the mutant polyglutamine-expanded AR and thereby ameliorates motor function of a mouse model of SBMA. ${ }^{54}$ Clearance of mutant AR by increased protein degradation or manipulation of protein control system also represents an attractive therapeutic strategy. The complex of Hsp90-client proteins, such as AR, is stabilized when it is associated with p23, a co-chaperone interacting with Hsp90, whereas inhibition of Hsp90 facilitates proteasomal degradation of its client proteins by altering the components of the protein complex. Treatment with 17-allylamino-17-demethoxygeldanamycin (17-AAG), a potent Hsp90 inhibitor, dissociates p23 from the Hsp90-AR complex, and thus facilitates proteasomal degradation of mutant AR in cellular and mouse models of SBMA. ${ }^{55}$

Geranylgeranylacetone, an acyclic isoprenoid compound with a retinoid skeleton, upregulates the levels of Hsp70 in the central nervous system and inhibits nuclear accumulation of mutant AR protein, resulting in amelioration of the neuromuscular phenotype of SBMA mice. ${ }^{56}$ Recent studies have also shown that alterations in the normal properties of the mutant protein are determinant for the disease pathogenesis. AR coregulators, such as ARA70, are alternative therapeutic targets, because they control the function and cellular distribution of AR. Curcumin-related compound 5-hydroxy-1, 7-bis (3,4-dimethoxyphenyl)-1,4,6-heptarine-3-one (ASJ-J9) was found to ameriolate the disease phenotype by disrupting the interaction between the AR and its co-regulator ARA70. ${ }^{57}$

Although there is no established therapy for SBMA, the experience of clinical trials has provided important insights into trial design, including outcome measures, biomarkers, and placebo effects in SBMA. Many compounds have shown efficacy in preclinical studies in mice, and the next step is to translate these findings into safe and effective treatment in patients. Well-designed clinical trials integrating validated 
Hashizume et al. 16

biomarkers and international collaboration are key to the successful development of disease-modifying therapies for SBMA. 


\section{References}

1. Katsuno M, Tanaka F, Adachi H, et al., Pathogenesis and therapy of spinal and bulbar muscular atrophy (SBMA). Prog Neurobiol 2012; 99: 246-56.

2. Atsuta N, Watanabe H, Ito M, et al., Natural history of spinal and bulbar muscular atrophy (SBMA): a study of 223 Japanese patients. Brain 2006; 129: 1446-55.

3. Hijikata Y, Hashizume A, Yamada S, et al., Biomarker-based analysis of preclinical progression in spinal and bulbar muscular atrophy. Neurology 2018; 90 : e1501-e1509.

4. Guber RD, Takyar V, Kokkinis A, et al., Nonalcoholic fatty liver disease in spinal and bulbar muscular atrophy. Neurology 2017; 89: 2481-2490.

5. Banno H, Katsuno M, Suzuki K, et al., Phase 2 trial of leuprorelin in patients with spinal and bulbar muscular atrophy. Ann Neurol 2009; 65:140-50.

6. Hashizume A, Katsuno M, Suzuki K, et al., Long-term treatment with leuprorelin for spinal and bulbar muscular atrophy: natural history-controlled study. J Neurol Neurosurg Psychiatry 2017; 88: 1026-1032.

7. Katsuno M, Banno H, Suzuki K, et al., Efficacy and safety of leuprorelin in patients with spinal and bulbar muscular atrophy (JASMITT study): a multicentre, randomised, double-blind, placebo-controlled trial. Lancet Neurol 2010; 9: 875-84.

8. Fernández-Rhodes LE, Kokkinis AD, White MJ, et al., Efficacy and safety of dutasteride in patients with spinal and bulbar muscular atrophy: a randomised placebo-controlled trial. Lancet Neurol 2011; 10: 140-7.

9. Querin G, D'Ascenzo C, Peterle E, et al., Pilot trial of clenbuterol in spinal and bulbar muscular atrophy. Neurology 2013; 80: 2095-8.

10. Grunseich C, Miller R, Swan T, et al., Safety, tolerability, and preliminary efficacy of an IGF-1 mimetic in patients with spinal and bulbar muscular atrophy: a randomised, placebo-controlled trial. Lancet Neurol 2018; 17: 1043-1052.

11. Querin G, Bede P, Marchand-Pauvert V, et al. Biomarkers of spinal and bulbar muscle atrophy (SBMA): A comprehensive review. Front Neurol 2018; 9: 844.

12. Hashizume A, Katsuno M, Suzuki K, et al. A functional scale for spinal and bulbar muscular atrophy: Cross-sectional and longitudinal study. Neuromuscul Disord 2015; 25: 554-62.

13. Dahlqvist JR, Fornander F, de Stricker Borch J, et al. Disease progression and 
outcome measures in spinobulbar muscular atrophy. Ann Neurol 2018;84:754-65.

14. Mano T, Katsuno M, Banno H, et al. Tongue pressure as a novel biomarker of spinal and bulbar muscular atrophy. Neurology 2014; 82: 255-62.

15. Klickovic U, Zampedri L, Sinclair CDJ, et al. Skeletal muscle MRI differentiates SBMA and ALS and correlates with disease severity. Neurology 2019; 93: e895-907.

16. Lehky TJ, Chen CJ, Di Prospero NA, et al. Standard and modified statistical MUNE evaluations in spinal-bulbar muscular atrophy. Muscle Nerve 2009; 40: 809-14.

17. Suzuki K, Katsuno M, Banno H, et al. The profile of motor unit number estimation (MUNE) in spinal and bulbar muscular atrophy. J Neurol Neurosurg Psychiatry 2010; 81, 567-71.

18. Lombardi V, Querin G, Ziff OJ, et al. Muscle and not neuronal biomarkers correlate with severity in spinal and bulbar muscular atrophy. Neurology 2019; 92: e1205-11.

19. Hijikata Y, Katsuno M, Suzuki K, et al. Impaired muscle uptake of creatine in spinal and bulbar muscular atrophy. Ann Clin Transl Neurol 2016; 3: 537-46.

20. La Spada AR, Wilson EM, Lubahn DB, et al. Androgen receptor gene mutations in X-linked spinal and bulbar muscular atrophy. Nature 1991; 352: 77-9.

21. Gardiner SL, Boogaard MW, Trompet S, et al. Prevalence of carriers of intermediate and pathological polyglutamine disease-associated alleles among large population-based cohorts. JAMA Neurol 2019; 76: 650-6.

22. Grunseich C, Kats IR, Bott LC, et al. Early onset and novel features in a spinal and bulbar muscular atrophy patient with a 68 CAG repeat. Neuromusc Disord 2014; 24: $978-81$.

23. Madeira JLO, Souza ABC, Cunha FS, et al. A severe phenotype of Kennedy disease associated with a very large CAG repeat expansion. Muscle Nerve. 2018; 57: E95-E97.

24. Watanabe M, Abe K, Aoki M, et al., Mitotic and meiotic stability of the CAG repeat in the X-linked spinal and bulbar muscular atrophy gene. Clin Genet 1996; 50: 113-137.

25. Tan MH, Li J, Xu HE, et al. Androgen receptor: structure, role in prostate cancer 
and drug discovery. Acta Pharmacol Sin. 2015; 36: 3-23.

26. Katsuno M, Adachi H, Kume A, et al. Testosterone reduction prevents phenotypic expression in a transgenic mouse model of spinal and bulbar muscular atrophy. Neuron 2002; 35: 843-54.

27. Zboray L, Pluciennik A, Curtis D, et al. Preventing the androgen receptor N/C interaction delays disease onset in a mouse model of SBMA. Cell Rep 2015; 13 : 2312-23.

28. Eftekharzadeh B, Banduseela VC, Chiesa G, et al. Hsp70 and Hsp40 inhibit an inter-domain interaction necessary for transcriptional activity in the androgen receptor. Nat Commun 2019; 10: 3562.

29. Nedelsky NB, Pennuto M, Smith RB, et al. Native functions of the androgen receptor are essential to pathogenesis in a Drosophila model of spinobulbar muscular atrophy. Neuron 2010; 67: 936-52.

30. Badders NM, Korff A, Miranda HC, et al. Selective modulation of the androgen receptor AF2 domain rescues degeneration in spinal bulbar muscular atrophy. Nat Med 2018; 24: 427-437.

31. Adachi H, Katsuno M, Minamiyama M, et al. Widespread nuclear and cytoplasmic accumulation of mutant androgen receptor in SBMA patients. Brain 2005; 128 : 659-70.

32. Iida M, Sahashi K, Kondo N, Nakatsuji H, et al. Src inhibition attenuates polyglutamine-mediated neuromuscular degeneration in spinal and bulbar muscular atrophy. Nat Commun 2019; 10: 4262.

33. Kondo N, Tohnai G, Sahashi K, et al. DNA methylation inhibitor attenuates polyglutamine-induced neurodegeneration by regulating Hes5. EMBO Mol Med 2019;11. pii: e8547.

34. Yamada S, Hashizume A, Hijikata Y, et al., Decreased peak expiratory flow associated with muscle fiber-type switching in spinal and bulbar muscular atrophy. PLoS One 2016; 11: e0168846.

35. Sorarù G, D'Ascenzo C, Polo A, et al., Spinal and bulbar muscular atrophy: skeletal muscle pathology in male patients and heterozygous females. J Neurol Sci 2008; 264: 100-5. 
36. Harding AE, Thomas PK, Baraitser M, et al., X-linked recessive bulbospinal neuronopathy: a report of ten cases. J Neurol Neurosurg Psychiatry 1982; 45: 1012-9.

37. Rocchi A, Milioto C, Parodi S, et al., Glycolytic-to-oxidative fiber-type switch and mTOR signaling activation are early-onset features of SBMA muscle modified by high-fat diet. Acta Neuropathol 2016; 132: 127-44.

38. Borgia D, Malena A, Spinazzi M, et al., Increased mitophagy in the skeletal muscle of spinal and bulbar muscular atrophy patients. Hum Mol Genet 2017; 26: 1087-1103.

39. Poort JE, Rheuben MB, Breedlove SM, et al., Neuromuscular junctions are pathological but not denervated in two mouse models of spinal bulbar muscular atrophy. Hum Mol Genet 2016; 25: 3768-3783.

40. Dahlqvist JR, Oestergaard ST, Poulsen NS, et al., Muscle contractility in spinobulbar muscular atrophy. Sci Rep 2019; 9: 4680.

41. Rhodes LE, Freeman BK, Auh S, et al. Clinical features of spinal and bulbar muscular atrophy. Brain 2009;132: 3242-51.

42. Chivet M, Marchioretti C, Pirazzini M, et al., Polyglutamine-expanded androgen receptor alteration of skeletal muscle homeostasis and myonuclear aggregation are affected by sex, age and muscle metabolism. Cells 2020; 9. pii: E325.

43. Cortes CJ, Ling SC, Guo LT, et al. Muscle expression of mutant androgen receptor accounts for systemic and motor neuron disease phenotypes in spinal and bulbar muscular atrophy. Neuron 2014;82:295-307.

44. Chua JP, Reddy SL, Merry DE, et al. Transcriptional activation of TFEB/ZKSCAN3 target genes underlies enhanced autophagy in spinobulbar muscular atrophy. Hum Mol Genet 2014;23:1376-86.

45. Yu Z, Wang AM, Adachi $\mathrm{H}$, et al. Macroautophagy is regulated by the UPR-mediator CHOP and accentuates the phenotype of SBMA mice. PLoS Genet 2011;7:e1002321.

46. Ranganathan S1, Harmison GG, Meyertholen K, et al., Mitochondrial abnormalities in spinal and bulbar muscular atrophy. Hum Mol Genet 2009; 18: $27-42$. 
47. Chivet M, Marchioretti C, Pirazzini M, et al., Polyglutamine-expanded androgen receptor disrupts muscle triad, calcium dynamics and the excitation-contraction coupling gene expression program bioRxiv 2019. doi: https://doi.org/10.1101/618405.

48. Cortes CJ, Ling SC, Guo LT, et al., Muscle expression of mutant androgen receptor accounts for systemic and motor neuron disease phenotypes in spinal and bulbar muscular atrophy. Neuron 2014; 82: 295-307.

49. Milioto C, Malena A, Maino E, et al., Beta-agonist stimulation ameliorates the phenotype of spinal and bulbar muscular atrophy mice and patient-derived myotubes. Sci Rep 2017; 7: 41046.

50. Palazzolo I, Stack C, Kong L, et al., Overexpression of IGF-1 in muscle attenuates disease in a mouse model of spinal and bulbar muscular atrophy. Neuron 2009; 63: 316-28.

51. Sobue G, Hashizume Y, Mukai E, et al. X-linked recessive bulbospinal neuronopathy. A clinicopathological study. Brain 1989; 112: 209-32.

52. Lieberman AP, Yu Z, Murray S, et al. Peripheral androgen receptor gene suppression rescues disease in mouse models of spinal and bulbar muscular atrophy. Cell Rep 2014; 7: 774-84.

53. Sahashi K, Katsuno M, Hung G, et al. Silencing neuronal mutant androgen receptor in a mouse model of spinal and bulbar muscular atrophy. Hum Mol Genet 2015; 24: 5985-94.

54. Miyazaki Y, Adachi H, Katsuno M, et al., Viral delivery of miR-196a ameliorates the SBMA phenotype via the silencing of CELF2. Nat Med 2012; 18: 1136-41.

55. Waza M, Adachi H, Katsuno M, et al., 17-AAG, an Hsp90 inhibitor, ameliorates polyglutamine-mediated motor neuron degeneration. Nat Med 2005; 11: 1088-95.

56. Katsuno M, Sang C, Adachi H, et al., Pharmacological induction of heat-shock proteins alleviates polyglutamine-mediated motor neuron disease. Proc Natl Acad Sci U S A 2005; 102: 16801-6.

57. Yang Z, Chang YJ, Yu IC, et al., ASC-J9 ameliorates spinal and bulbar muscular atrophy phenotype via degeneration of androgen receptor. Nat Med 2007; 13 : 348-53. 


\section{Figure Legends}

\section{Figure 1. Clinical course of SBMA}

The onset of muscle weakness is between 30 and 60 years in most patients with SBMA. Decrease in serum creatinine levels precedes the onset of subjective muscle weakness by more than 15 years, followed by the emergence of hand tremor. Health checkup data of patients also suggests that serum levels of liver enzymes, and possibly creatine kinase $(\mathrm{CK})$, begin to increase around 10 years before the onset of weakness. On the other hand, subjective dysphagia starts around 10 years after the onset, eventually resulting in aspiration pneumonia.

\section{Figure 2. Molecular pathogenesis of SBMA}

Upon ligand binding, the polyglutamine-expanded AR (androgen receptor) is dissociated from molecular chaperones, undergoes N/C interaction, and translocates into the nucleus. In the nucleus, the pathogenic AR dimerizes and forms aggregates that require DNA binding and interaction with co-activators. The accumulated AR instigates several abnormal events, such as protein hyperphosphorylation and epigenetic dysregulation. NTD, N-terminal domain; DBD, DNA binding domain; LBD, ligand binding domain. 
Table 1. Clinical trials of candidate drugs for SBMA

\begin{tabular}{|c|c|c|c|c|c|}
\hline & $\begin{array}{l}\text { Number } \\
\text { of } \\
\text { Subjects }\end{array}$ & Interventions & $\begin{array}{l}\text { Follow-up } \\
\text { period }\end{array}$ & $\begin{array}{l}\text { Primary } \\
\text { outcomes }\end{array}$ & $\begin{array}{l}\text { Principal secondary } \\
\text { outcomes }\end{array}$ \\
\hline $\begin{array}{l}\text { Banno et } \\
\text { al., } 2006\end{array}$ & 50 & $\begin{array}{l}\text { leuprorelin } \\
\text { acetate }\end{array}$ & 48 weeks & ALSFRS-R & $\begin{array}{l}\text { Video fluorography } \\
\text { Anti-polyglutamine } \\
\text { immunostaining of the } \\
\text { scrotal skin }\end{array}$ \\
\hline $\begin{array}{l}\text { Katsuno et } \\
\text { al., } 2010\end{array}$ & 204 & $\begin{array}{l}\text { leuprorelin } \\
\text { acetate }\end{array}$ & 48 weeks & $\begin{array}{l}\text { Pharyngeal } \\
\text { barium residue } \\
\text { in video } \\
\text { fluorography }\end{array}$ & $\begin{array}{l}\text { Anti-polyglutamine } \\
\text { immunostaining of the } \\
\text { scrotal skin }\end{array}$ \\
\hline $\begin{array}{l}\text { Rhodes et } \\
\text { al., } 2011\end{array}$ & 50 & dutasteride & 24 months & QMA & $\begin{array}{l}\text { Serum creatine kinase } \\
\text { Muscle strength }\end{array}$ \\
\hline $\begin{array}{l}\text { Querin et } \\
\text { al., } 2013\end{array}$ & 20 & clenbuterol & 12 months & $\begin{array}{l}\text { 6-minute } \\
\text { walking distance }\end{array}$ & $\begin{array}{l}\text { MRC scale } \\
\text { ALSFRS-R }\end{array}$ \\
\hline $\begin{array}{l}\text { Grunseich } \\
\text { et al. } 2018\end{array}$ & 27 & $\begin{array}{l}\text { IGF-1 } \\
\text { mimetic } \\
(\mathrm{BVS} 857)\end{array}$ & 12 weeks & TMV & AMAT \\
\hline
\end{tabular}

ALSFRS-R; the Revised Amyotrophic Lateral Sclerosis Functional Rating Scale: QMA; quantitative muscle assessment: MRC scale; Medical Research Council scale: TMV; thigh muscle volume: AMAT; the Adult Myopathy Assessment Tool 
Years from onset of weakness

Decrease in serum creatinine level

Hand tremor

Elevation of liver enzyme

Subjective muscle weakness

i

I 


\section{Androgen receptor}

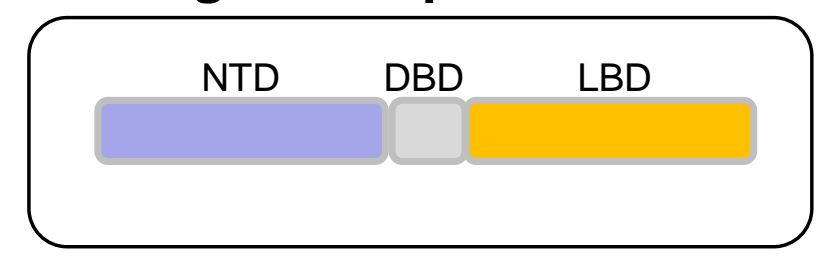

Polyglutamine tract

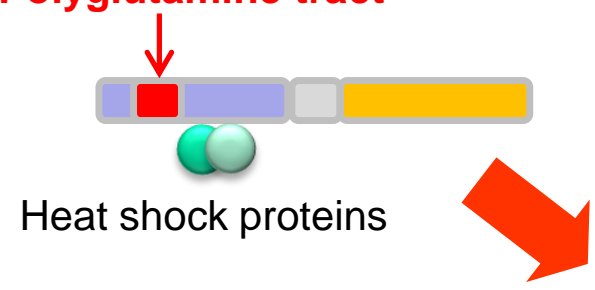

$$
\text { Androgens }
$$

Cytoplasm

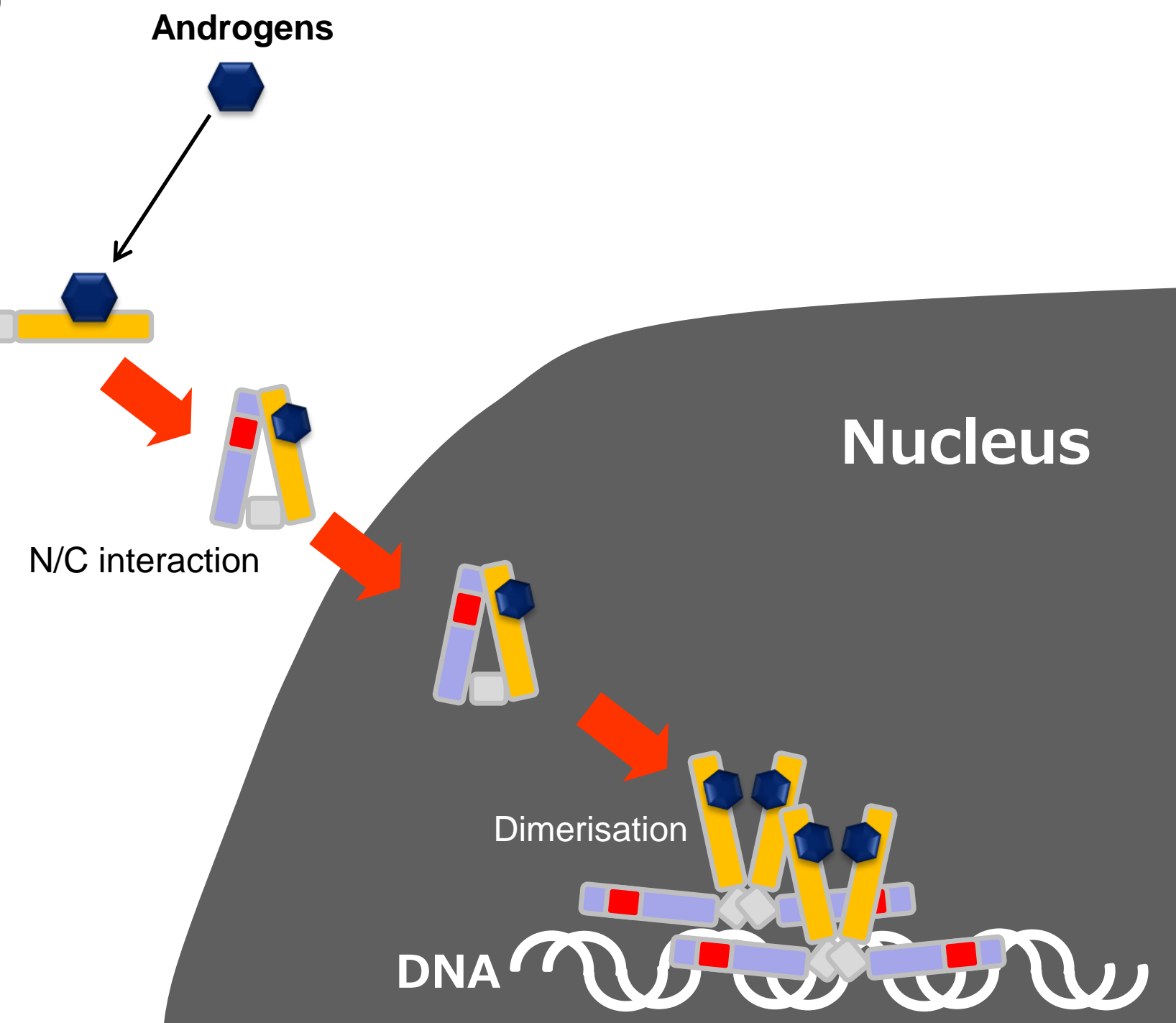

